\title{
UNIVERSITYOF
}

FORWARD

THINKING

WESTMINSTER用

WestminsterResearch

http://www.westminster.ac.uk/westminsterresearch

\section{Simulating Business Processes of Manufacturing SMEs on the}

Cloud

Terstyanszky, G., Kiss, T., Ozkok, A and Isler, V

This is a copy of the published version of Terstyanszky G, Kiss T, Ozkok A, Isler V. (2016) Simulating business processes of manufacturing SMEs on the cloud. PeerJ Preprints 4:e2509v1 https://doi.org/10.7287/peerj.preprints.2509v1

(C) 2016 Terstyanszky et al.

Licence: This is an open access article distributed under the terms of the Creative Commons Attribution License, which permits unrestricted use, distribution, reproduction and adaptation in any medium and for any purpose provided that it is properly attributed. For attribution, the original author(s), title, publication source (PeerJ Preprints) and either DOI or URL of the article must be cited.

The WestminsterResearch online digital archive at the University of Westminster aims to make the research output of the University available to a wider audience. Copyright and Moral Rights remain with the authors and/or copyright owners.

Whilst further distribution of specific materials from within this archive is forbidden, you may freely distribute the URL of WestminsterResearch: ((http://westminsterresearch.wmin.ac.uk/).

In case of abuse or copyright appearing without permission e-mail repository@westminster.ac.uk 


\title{
Simulating Business Processes of Manufacturing SMEs on the Cloud
}

\author{
Gabor Terstyanszky, Tamas Kiss \\ Center for Parallel Computing, University of Westminster, London, UK \\ G.Z.Terstyanszky@westminster.ac.uk \\ Ahmet Özkök, Veysi İșler \\ Simsoft Bilgisayar Teknolojileri Ltd, Ankara, Turkey \\ ahmet.ozkok@simsoft.com.tr
}

\begin{abstract}
Simulating the efficiency of business processes could reveal crucial bottlenecks for manufacturing companies and could lead to significant optimizations resulting in decreased time to market, more efficient resource utilization, and larger profit. While such business optimization software is widely utilized by larger companies, SMEs typically do not have the required expertise and resources to efficiently exploit these advantages. The aim of this work is to explore how simulation software vendors and consultancies can extend their portfolio to SMEs by providing business process optimization based on a cloud computing platform. By executing simulation runs on the cloud, software vendors and associated business consultancies can get access to large computing power and data storage capacity on demand, run large simulation scenarios on behalf of their clients, analyze simulation results, and advise their clients regarding process optimization. The solution is mutually beneficial for both vendor/consultant and the end-user SME. End-user companies will only pay for the service without requiring large upfront costs for software licenses and expensive hardware. Software vendors can extend their business towards the SME market with potentially huge benefits.
\end{abstract}

SimBusPro [1], a product of SimSoft Ltd [2], is a commercial business process modelling, simulation and optimization software package based on the BPMN2 standard. To run a business process simulation, SimBusPro first creates start events to define tokens that represent customer demands, and adds them to the simulation model. Next, it runs service tasks to simulate the production processes. Finally, it invokes end tasks to stop simulation and remove tokens from the simulation model. SimBusPro can run parameter study type simulations where business processes have different sets of input parameters for different models producing a set of simulation results that can be compared and evaluated to select the best model(s). SimBusPro can optimize the selected simulation models using different optimization techniques such as genetic algorithms or tabu search.

The European CloudSME project [3] developed a generic platform and its associated methodology that enable the efficient migration of simulation applications to the cloud. Using the CloudSME simulation platform [4] simulation software products, such as SimBusPro, can be easily extended with cloud support.

SimBusPro has three major components: a Simulation Application, an Optimization Application and a Desktop Application. The Simulation and the Optimization applications are Java executables which are deployed on cloud resources. The Desktop Application interacts with these clouds using the CloudBroker Platform and its API [5]. The Optimization Application takes the business process model file as input from the Desktop Application and creates and sends alternative models separately to the Simulation Application. It also collects results, applies decisions, generates one final single model, which is the optimized model, and sends this model back to the Desktop Application.

The cloud extended SimBusPro software has been successfully demonstrated in two SME case-studies. The business processes of two Turkish SMEs, Gokdogan and Ozdekan have been successfully simulated and analyzed. Based on the outcome of this analysis, suggestions have been made to both companies for optimizing their processes. Neither of these companies has used simulation solutions in the past and could not even consider utilizing such solutions due to their complexity and high costs. However, based on the cloud-based simulation solution, SimSoft can now offer affordable consultancy based on scalable and on-demand simulation runs.

\section{ACKNOWLEDGMENT}

The research leading to these results has received funding from the CloudSME project, supported by the European Commission Seventh Framework Programme (FP7) (grant agreement no. 60886). 
$8^{\text {th }}$ International Workshop on Science Gateways (IWSG 2016), 8-10 June 2016 sc

\section{REFERENCES}

1. SimBusPro - http://simbuspro.com

2. Simsoft - http://www.simsoft.com.tr/en

3. CloudSME project - http://www.cloudsme.eu

4. S. J. Taylor, T. Kiss, G. Terstyanszky, P. Kacsuk, N. Fantini: Cloud Computing for Simulation in Manufacturing and Engineering: Introducing the CloudSME Simulation Platform, ANSS'14, Proceedings of the 2014 Annual Simulation Symposium, Society for Computer Simulation International, Article No. 12

5. W. Sudholt: HPC as a SaaS: The CloudBroker Solution, Swiss Distributed Computing Day, University of Bern, 28 Nov. 2011 